\title{
Idiopathic Granulomatous Lobular Mastitis in a Male Breast: A Case
}

\section{Report}

\author{
Leehi Joo, ${ }^{1}$ Soo Hyun Yeo, ${ }^{1,}$ and Sun Young Kwon ${ }^{2}$ \\ ${ }^{1}$ Department of Radiology, Dong-San Medical Center, Keimyung University College of Medicine, Daegu, Korea \\ ${ }^{2}$ Department of Pathology, Dong-San Medical Center, Keimyung University College of Medicine, Daegu, Korea \\ 'Corresponding author: Soo Huyn Yeo, Department of Radiology, Dong-San Medical Center, Keimyung University College of Medicine, 56 Dalseung-Ro, Jung-Gu, Daegu, 41931, \\ Korea. Tel: +82-532507770, Fax: +82-532507766, E-mail: shyeo81@hanmail.net
}

Received 2017 June 23; Revised 2017 November 29; Accepted 2018 January 14.

\begin{abstract}
Idiopathic granulomatous lobular mastitis (IGLM) that mimics breast cancer both clinically and radiologically is a chronic inflammatory condition of the breast without a known etiology. It usually affects childbearing women and is associated with pregnancy, lactation, or use of oral contraceptives. IGLM in a male breast is extremely rare, and only two case reports have been published. A 60-year-old man was referred to our hospital for right breast mass. He had right breast pain with a small palpable lump for 2 weeks. Ultrasonography (US) was performed with color Doppler US and US elastography. The lesion was diagnosed as IGLM pathologically by 14 gauge core needle biopsy. We describe a very rare case of IGLM arising from a male breast based on ultrasonographic and pathologic findings. IGLM should be considered as a differential diagnosis in male breast diseases, although the imaging findings may not be comparable with typical IGLM.
\end{abstract}

Keywords: Granulomatous Mastitis, Breast Neoplasms, Male

\section{Introduction}

Male breast tissue is similar to female breast tissue before puberty. However, during puberty, the male breast develops differently compared to its female counterpart as a result of androgenic effects (1). Anatomically, the male breast is composed of a small nipple, areola, and subcutaneous fat tissue containing few residual ducts and stroma in contrast with the female breast, which consists of mainly fibroglandular tissue with ducts, stroma, and fat tissue $(2,3)$. Histologically, few subareolar ducts of the breast elongate and branch under the stimulation of hormone or drugs. The Lobule and Cooper's ligament is not visible in the normal male breast $(3,4)$ Since terminal ductal lobular units where most diseases of the female breast occur do not develop in the male breast, male breast diseases occur within skin, fat, stroma, glands, neurovascular structures, and lymphatic vessels (1).

Idiopathic granulomatous lobular mastitis (IGLM) is a rare chronic inflammatory disease without an obvious etiology, and it is characterized by non-necrotizing granuloma formation affecting mammary lobules with microabscesses and without evidence of a microorganism (5-7). As IGLM affects mainly the mammary lobule, IGLM in male patients is extremely rare, and only two cases have been re- ported so far as a case report and brief report $(8,9)$.

Here we describe a very rare case of IGLM in the male breast based on ultrasonographic and pathologic findings.

\section{Case Presentation}

A 60-year-old man was referred to our hospital for further evaluation of a right breast mass. He had right breast pain with a small palpable lump for 2 weeks. He did not have any systemic disease or specific infection, such as tuberculosis. He visited a local medical center for evaluation of a right breast lesion. Ultrasonography (US) of the right breast was performed using the AplioTM 500 platinum US system (Toshiba Medical System Corporation, Otawara, Tochigi, Japan), which was equipped with a $12-6.2 \mathrm{MHz}$ linear-arrary transducer. Ultrasonogram exhibited a 12mm oval, circumscribed, hypoechoic mass in the subareolar portion of the right breast. He was referred to our hospital for pathologic confirmation using biopsy to differentiate breast cancer from benign breast disease, such as nodular gynecomastia or a breast abscess. A scant amount of fibroglandular breast tissue was observed in both breasts, which was diagnosed as gynecomastia without other relevant clinical findings. On physical examination, the bean- 
sized palpable lesion was noted in the subareolar portion of the right breast with nipple retraction. Inflammatory signs, such as erythematous change or warm skin were not found. Initial laboratory findings revealed a white blood cell count and C-reactive protein level within normal limits. The ultrasonogram performed at our hospital showed a $12 \times 7-\mathrm{mm}$ oval, circumscribed, hypoechoic mass in the subareolar portion of the right breast, corresponding to the palpable site on the patient (Figure 1). The mass showed hypervascularity on the color Doppler examination, as peripheral and internal vascularity according to the breast imaging reporting and data system (BIRADS) Fifth Edition classification system. Additionally, USelastography of the mass was performed, and soft stiffness was noted in the right subareolar mass, according to the BI-RADS Fifth Edition classification system. The lesion was assessed as BI-RADS category $4 \mathrm{~b}$, and US-guided core needle biopsy was performed using a 14-gauge needle. We obtained five biopsy cores, and the lesion was confirmed pathologically as granulomatous lobular mastitis (Figure 2 ). The patient was treated with corticosteroids, and the lesion decreased in size clinically by the 3-month follow-up.

\section{Discussion}

Male breast diseases are most often benign (10). Gynecomastia is the most common benign breast pathology in male breasts, with a reported prevalence of approximately $4 \%$ in the age group of $10-19$ years, and it has been found in up to $55 \%$ of male breasts in one autopsy specimen series (10). Mastitis, a benign breast inflammation, is exceedingly rare in men with only three cases reported in the literature to date as a case report or original article ( 9 , 11).

IGLM is a rare, benign inflammatory disease of the breast that mainly affects women of reproductive age (5, 7,12 ). IGLM is characterized by a chronic, lobular inflammatory process and noncaseating granulomatous inflammation without an obvious etiology. The diagnosis of IGLM can be made by excluding all known causes of granulomatous inflammation, such as mammary ductectasia, Wegener granuloma, sarcoidosis, tuberculosis, and histoplasmosis (12).

The etiology of IGLM is unclear, but an autoimmune origin is the most widely accepted theory because of the response to steroid therapy (5). Bani-Hani et al. (12) identified four possible mechanisms of IGLM, and its association with lactation or hyperprolactinemia is explained by extravasated lactational secretions damaging the ductal epithelium and leading to a granulomatous inflammatory response. Additionally, oral contraceptives can cause granulomatous lobular mastitis by a chemical reaction. Our patient did not have any hyperprolactinemic condition caused by a pituitary tumor or drug-induced galactorrhea.

IGLM develops mostly in women of reproductive age (mean age: 33.1 - 39.5 years, range: 22 - 59 years) and women with children, who have a history of oral contraceptive use and/or lactation $(5-7,13)$. The most common symptom is a palpable mass, followed by pain, swelling, erythema, and axillary lymphadenopathy. In IGLM, nipple retraction rarely occurs in women, but it usually occurs in $40-50 \%$ of men with breast cancer (14). Inflammatory signs may not always be present clinically; thus, a misdiagnosis of breast cancer can be made (5).

Imaging features of IGLM in female patients have been reported infrequently since IGLM was first described by Kessler et al. in 1972 and recently reviewed in several reports of small series. There is a wide spectrum of radiologic findings of IGLM because of various imaging modalities, such as mammography, US, and magnetic resonance imaging $(4,7,15,16)$. Focal asymmetric densities were the most frequent mammographic finding, followed by an irregular mass in most case series or studies of IGLM. Microcalcifications were not seen on any mammograms in our patient with IGLM. According to several reports, the most frequent sonographic finding is an irregular, heterogeneous hypoechoic mass with tubular extension $(7,15,16)$. Some studies of color Doppler US in patients with IGLM reported increased intralesional and perilesional vascularity $(15,17)$. US-elastography is widely used in breast imaging. The differentiation between IGLM and breast malignancy has been studied using radiation force impulse imaging, i.e., acoustic radiation force impulse (ARFI) elastography (13), and the authors revealed that IGLM had lower shearwave velocity than breast malignancy. This indicated that IGLM was softer than a malignancy, and a higher diagnostic accuracy was achieved using ARFI combined with US in this retrospective study.

However, imaging findings of IGLM could not been fully differentiated from those of breast malignancy on a mammogram or ultrasonogram $(3,4,7,10,16)$. A definitive diagnosis of IGLM should be made histologically from biopsy results because clinical features and imaging findings are not specific and similar to those of breast cancer.

The aforementioned clinical features and representative imaging features of IGLM were obtained from studies with female patients with IGLM. These features of IGLM have not been studied in male patients so far.

In the present case, ultrasonographic findings showed an oval, circumscribed, hypoechoic mass in the subareolar portion of the right breast. On a color Doppler ultrasonogram, perilesional and internal vascularity was noted. Furthermore, soft elasticity, according to BI-RADS Fifth Edition classification system, was observed using US-elastography. 

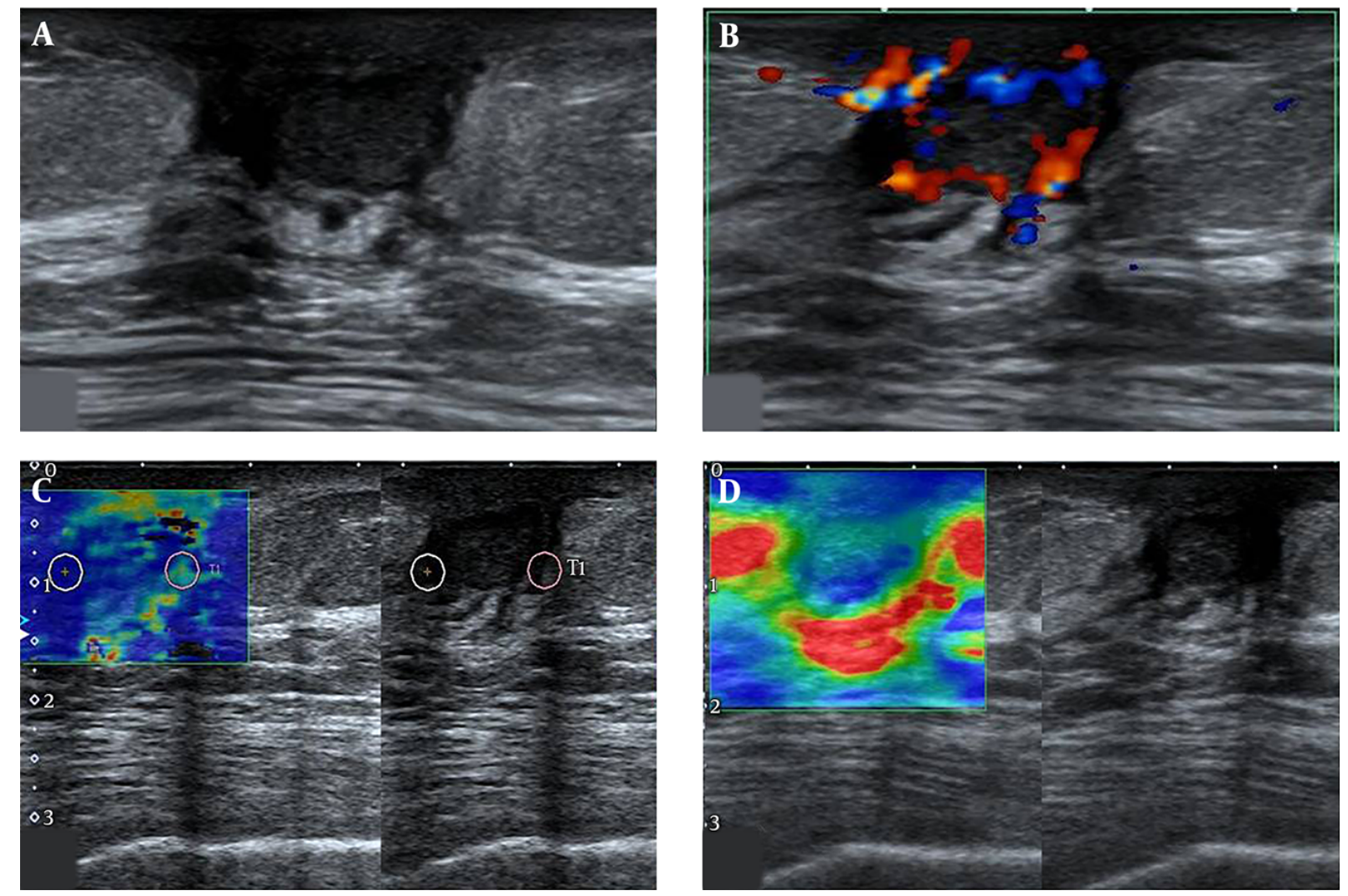

Figure 1. Ultrasonographic images of idiopathic granulomatous lobular mastitis in a 60-year old male patient. A, Ultrasonography of the right breast reveals $12 \times 7$ mm sized, oval shape, relatively circumscribed margin, hypoechoic mass at subareolar portion of the right breast; B, Color Doppler US of right breast mass reveals increased perilesional and intralesional vascularity at right subareolar mass, assessed as color Doppler score 3 according to breast imaging reporting and data system (BI-RADS) fifth edition; C, Strain elastography of right breast mass reveals mixed blue and green color mapping at the mass, soft elasticity according to BI-RADS fifth edition; D, Shear-wave elastography of right breast mass reveals peripheral green-yellow color mapping, showing $38 \mathrm{kPa}$ as a quantitative scale.
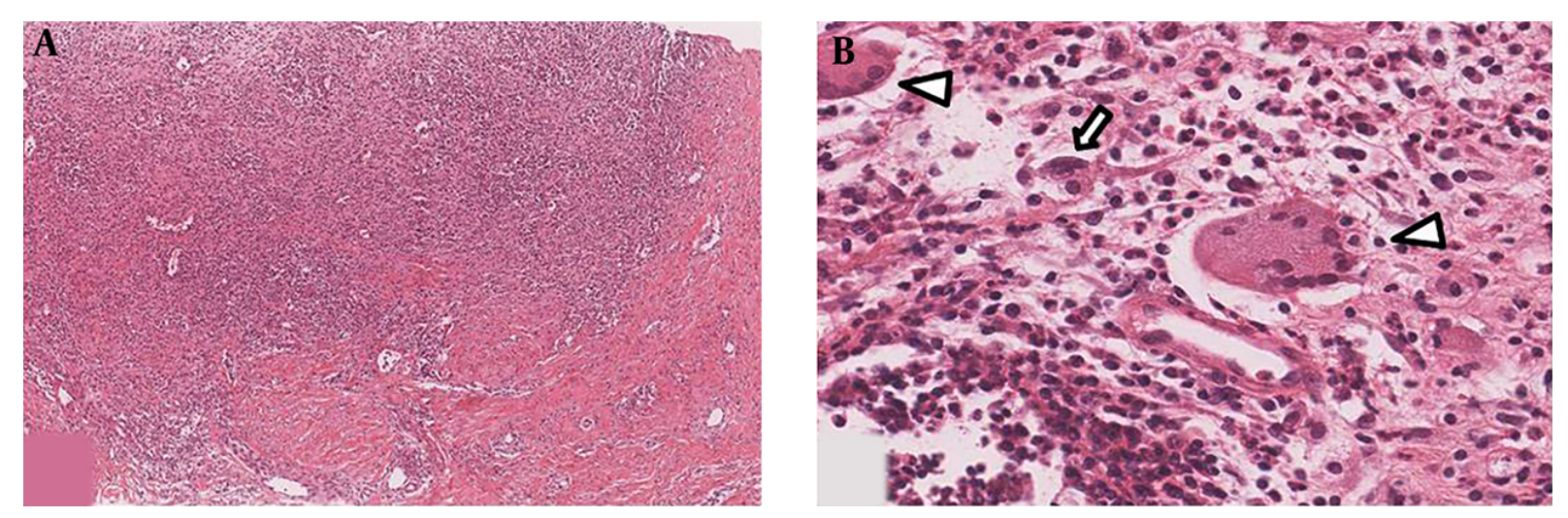

Figure 2. Histopathology of the breast biopsy specimen. A, Low power field of microscopic finding of the biopsy reveals a heavy infiltration of inflammatory cells that has destroyed much of lobules (H\&E staining, $\times 40$ ); B, Granulomas are composed of epithelioid histiocytes (arrow) and Langerhans giant cells (arrowheads) accompanied by lymphocytes, plasma cells, and occasional eosinophils (H\&E staining, $\times 200)$.

The sonographic finding of our patient is not comparable with the typical sonographic finding of IGLM reported in past studies. Two case reports of IGLM in men did not publish mammograms or sonograms. Further study of the 
imaging features of IGLM in male patients is needed. To the best of our knowledge, this is the first report of IGLM arising in a male breast with imaging studies of ultrasonography, including color Doppler US and US-elastography.

In conclusion, IGLM rarely occurs in male breasts, although the lobule is not developed in male breasts and the imaging features on a mammogram or ultrasonogram are not comparable to that of female IGLM. When a palpable breast mass occurs in a man, physicians must consider male breast diseases ranging from benign diseases, such as gynecomastia, to breast cancer. Moreover, inflammatory breast diseases such as IGLM should be considered as a differential diagnosis, although the imaging findings may not be comparable with typical IGLM.

\section{Acknowledgments}

None.

\section{Footnotes}

Authors' Contributions: Soo Hyun Yeo developed the original idea, abstracted, wrote the manuscript, and is guarantor. Leehi Joo contributed to prepare figures of ultrasonography and prepared the manuscript. Sun Young Kwon contributed to prepare figures of histopathology and prepared the manuscript.

\section{Conflicts of Interests: None.}

Financial Disclosure: None.

Funding/Support: None.

\section{References}

1. Lattin GJ, Jesinger RA, Mattu R, Glassman LM. From the radiologic pathology archives: diseases of the male breast: radiologicpathologic correlation. Radiographics. 2013;33(2):461-89. doi: 10.1148/rg.332125208. [PubMed: 23479708].

2. Iuanow E, Kettler M, Slanetz PJ. Spectrum of disease in the male breast. AJR Am J Roentgenol. 2011;196(3):W247-59. doi: 10.2214/AJR.09.3994. [PubMed: 21343472].

3. Adibelli ZH, Oztekin O, Gunhan-Bilgen I, Postaci H, Uslu A, Ilhan E. Imaging characteristics of male breast disease. Breast J. 2010;16(5):510-8. doi: 10.1111/j.1524-4741.2010.00951.x. [PubMed: 20560973].
4. Yitta S, Singer CI, Toth HB, Mercado CL. Image presentation. Sonographic appearances of benign and malignant male breast disease with mammographic and pathologic correlation. J Ultrasound Med 2010;29(6):931-47. [PubMed: 20498468].

5. Sripathi S, Ayachit A, Bala A, Kadavigere R, Kumar S. Idiopathic granulomatous mastitis: a diagnostic dilemma for the breast radiologist. Insights Imaging. 2016;7(4):523-9. doi: 10.1007/s13244-016-0497-2. [PubMed: 27164916]. [PubMed Central: PMC4956625].

6. Gurleyik G, Aktekin A, Aker F, Karagulle H, Saglamc A. Medical and surgical treatment of idiopathic granulomatous lobular mastitis: a benign inflammatory disease mimicking invasive carcinoma. $J$ Breast Cancer. 2012;15(1):119-23. doi: 10.4048/jbc.2012.15.1.119. [PubMed: 22493638]. [PubMed Central: PMC3318163].

7. Hovanessian Larsen LJ, Peyvandi B, Klipfel N, Grant E, Iyengar G. Granulomatous lobular mastitis: imaging, diagnosis, and treatment.AJRAm JRoentgenol.2009;193(2):574-81. doi:10.2214/AJR.08.1528. [PubMed: 19620458].

8. Al Manasra AR, Al-Hurani MF. Granulomatous Mastitis: A Rare Cause of Male Breast Lump. Case Rep Oncol. 2016;9(2):516-9. doi: 10.1159/000448990. [PubMed: 27721777]. [PubMed Central: PMC5043282].

9. Reddy KM, Meyer CE, Nakdjevani A, Shrotria S. Idiopathic granulomatous mastitis in the male breast. Breast J. 2005;11(1):73. doi: 10.1111/j.1075-122X.2005.21404.x. [PubMed: 15647084].

10. Yen PP, Sinha N, Barnes PJ, Butt R, Iles S. Benign and Malignant Male Breast Diseases: Radiologic and Pathologic Correlation. Can Assoc Radiol J. 2015;66(3):198-207. doi: 10.1016/j.carj.2015.01.002. [PubMed: 26073217].

11. Korkut E, Akcay MN, Karadeniz E, Subasi ID, Gursan N. Granulomatous Mastitis: A Ten-Year Experience at a University Hospital. Eurasian J Med. 2015;47(3):165-73. doi: 10.5152/eurasianjmed.2015.118. [PubMed: 26644764]. [PubMed Central: PMC4659517].

12. Bani-Hani KE, Yaghan RJ, Matalka ,I, Shatnawi NJ. Idiopathic granulomatous mastitis: time to avoid unnecessary mastectomies. Breast J. 2004;10(4):318-22. doi: 10.1111/j.1075-122X.2004.21336.x. [PubMed: 15239790].

13. Teke M, Teke F, Alan B, Turkoglu A, Hamidi C, Goya C, et al. Differential diagnosis of idiopathic granulomatous mastitis and breast cancer using acoustic radiation force impulse imaging. J Med Ultrason (2001). 2017;44(1):109-15. doi: 10.1007/s10396-016-0749-2. [PubMed: 27787642]

14. Javidiparsijani S, Rosen LE, Gattuso P. Male Breast Carcinoma: A Clinical and Pathological Review. Int J Surg Pathol. 2017;25(3):200-5. doi: 10.1177/1066896916675953. [PubMed: 27831530].

15. Ozturk M, Mavili E, Kahriman G, Akcan AC, Ozturk F. Granulomatous mastitis: radiological findings. Acta Radiol. 2007;48(2):150-5. doi 10.1080/02841850601128975. [PubMed: 17354134].

16. Yilmaz E, Lebe B, Usal C, Balci P. Mammographic and sonographic findings in the diagnosis of idiopathic granulomatous mastitis. Eur Radiol. 2001;11(11):2236-40. doi: 10.1007/s003300100965. [PubMed: 11702165].

17. Engin G, Acunas G, Acunas B. Granulomatous mastitis: gray-scale and color Doppler sonographic findings. J Clin Ultrasound. 1999;27(3):1016. [PubMed: 10064406]. 